\title{
Erratum to: A manual-based vocational rehabilitation program for patients with an acquired brain injury: study protocol of a pragmatic randomized controlled trial (RCT)
}

Louise K. Hoeffding ${ }^{1,2,3}$, Maria Haahr Nielsen 1,3, Morten A. Rasmussen ${ }^{4,5}$, Anne Norup ${ }^{6}$, Juan Carlos Arango-Lasprilla ${ }^{7,8}$, Ulrikka K. Kjær ${ }^{9}$, Kristoffer Sølvsten Burgdorf ${ }^{1,3}$, Kirsten Jensen Quas ${ }^{10}$ and Trine Schow ${ }^{1 *}$

\section{Erratum}

The original publication [1] misses one author. The original article has been updated to rectify this error. All details about the missing author can be found below:

Kirsten Jensen Quas, Neuropsychologist, Head of Research and Development at Brain Injury Center BOMI, Roskilde, Denmark.

\begin{abstract}
Author details
'Research and development, Brain Injury Center BOMI, Maglegaardsvej 15, 4000 Roskilde, Denmark. ${ }^{2}$ Institute of Biological Psychiatry, Mental Health Centre Sct. Hans, Copenhagen University Hospital, Roskilde, Denmark. ${ }^{3}$ Department of Clinical Immunology, the Blood Bank, Rigshospitalet, University Hospital of Copenhagen, Copenhagen, Denmark. ${ }^{4}$ Faculty of Science, Chemometrics and Analytical Technology, University of Copenhagen, Copenhagen, Denmark. ${ }^{5}$ Copenhagen Prospective Studies on Asthma in Childhood, Faculty of Health and Medical Sciences, University of Copenhagen \& Danish Pediatric Asthma Center, Gentofte Hospital, University of Copenhagen, Copenhagen, Denmark. ${ }^{6}$ National Study on Young Brain Injury Survivors, Department of Neurology, Rigshospitalet-Copenhagen University Hospital, Copenhagen, Denmark. ${ }^{7}$ BioCruces Health Research Institute, Cruces University Hospital, Barakaldo, Spain. ${ }^{8}$ IKERBASQUE. Basque Foundation for Science, Bilbao, Spain. ${ }^{9}$ Kjaer Management, 3520 Farum, Denmark. ${ }^{10}$ Neuropsychologist, Head of Research and Development at Brain Injury Center BOMl, Roskilde, Denmark.
\end{abstract}

Published online: 06 September 2017

\section{Reference}

1. Hoeffding LK, Nielsen MH, Rasmussen MA, Norup A, Arango-Lasprilla JC,

Kjær UK, Burgdorf KS, Quas K, Schow T. A manual-based vocational rehabilitation program for patients with an acquired brain injury: study protocol of a pragmatic randomized controlled trial (RCT) Trials 2017 18:371 DOI: 10.1186/s13063-017-2115-0.

\footnotetext{
* Correspondence: Trineschow@bomi.roskilde.dk

'Research and development, Brain Injury Center BOMI, Maglegaardsvej 15,
} 4000 Roskilde, Denmark 